\title{
Fluctuation relations with intermittent non-Gaussian variables
}

\author{
Adrián A. Budini \\ Consejo Nacional de Investigaciones Científicas y Técnicas (CONICET), \\ Centro Atómico Bariloche, Avenida E. Bustillo Km 9.5, (8400) Bariloche, Argentina
}

(Dated: September 22, 2018)

\begin{abstract}
Non-equilibrium stationary fluctuations may exhibit a special symmetry called fluctuation relations (FR). Here, we show that this property is always satisfied by the subtraction of two random and independent variables related by a thermodynamic-like change of measure. Taking one of them as a modulated Poisson process, it is demonstrated that intermittence and FR are compatibles properties that may coexist naturally. Strong non-Gaussian features characterize the probability distribution and its generating function. Their associated large deviation functions (LDF) develop a "kink" at the origin and a plateau regime respectively. Application of this model in different stationary nonequilibrium situations is discussed.
\end{abstract}

PACS numbers: 05.70.Ln, 05.40.-a, 45.70.-n, 82.70.Dd

\section{INTRODUCTION}

Intermittency is a phenomenon that appears in a wide class of situations such as chaotic dynamics [1], atomic [2] and nanoscopic 3] fluorescent systems, single-molecule reaction dynamics [4], biological self-organized models [5], or fluid turbulence [6], just to name a few. It consists in a random switching amongst qualitatively different system dynamical regimes. Its stochastic behavior may be ergodic or not. Complexity, non-Gaussian statistics, and nonequilibrium dynamics are closely related with its development. In this last context, (Gallavoti-Cohen) FR 7] addresses the distribution of entropy production in far-from-equilibrium steady states. It relates the probability of observing a certain entropy production rate to the probability of observing the corresponding entropy consumption rate $7-12]$. Theoretical and experimental results confirm its validity in diverse nonequilibrium systems [13 22]. Interestingly, the symmetry imposed by FR has also been found valid for non-thermodynamic variables [23].

As FR and intermittence are intrinsically related with nonequilibrium dynamics, it is natural to ask about the possible coexistence of both properties. The main goal of this contribution is to give a positive answer to this issue. We demonstrate that there may exist non-equilibrium steady states whose (non-Gaussian) fluctuations are intermittent and satisfy the FR. An explicit construction of a stochastic variable with the required symmetry defines the basis of the present analysis. It is shown that FR can always be satisfied by the subtraction of two independent stochastic variables related by an exponential change of measure. Hence, intermittence is introduced by choosing one of them as a random modulated Poisson process. Special attention is paid to the asymptotic regime, where FR can be analyzed through the large deviation functions (LDF) [7 9] of the probability distribution and its associated generating function. In the parameter regime where intermittence arises, they develop a kink around the origin and a plateau regime respectively. These strong nonGaussian features are similar to that found in different observables such as the entropy production rate of a colloidal particle [22] and the (time-average) velocity of a polar granular rod [23]. While the specific analysis of these systems is beyond the scope of this contribution, the present results suggest that intermittence under the constraint of FR symmetries may be a central ingredient when studying nonequilibirum states characterized by non-Gaussian fluctuations.

\section{FLUCTUATION RELATIONS WITH INDEPENDENT STOCHASTIC VARIABLES}

Our results rely in the following analysis. Let an arbitrary stochastic variable $x_{s t}$, whose probability distribution $P(x)$ satisfies a FR defined as

$$
P(-x)=P(x) \exp [-\zeta x]
$$

where $\zeta$ is a given real positive constant. This symmetry implies that negative values are exponentially less probable than positive values. In terms of the generating function $Z(\lambda)=\left\langle e^{-\lambda x_{s t}}\right\rangle=\int_{-\infty}^{+\infty} d x P(x) e^{-\lambda x}, Z(0)=1$, the $\mathrm{FR}$ reads

$$
Z(\lambda)=Z(-\lambda+\zeta) .
$$

In order to satisfy the FR, we write $x_{s t}$ as the subtraction of two statistically independent variables $x_{s t}^{ \pm}$,

$$
x_{s t}=x_{s t}^{+}-x_{s t}^{-} .
$$

Then, $Z(\lambda)=Z_{+}(\lambda) Z_{-}(-\lambda)$, where $Z_{ \pm}(\lambda)$ are the generating functions of $x_{s t}^{ \pm}$, and whose distributions are $P_{ \pm}(x)$. The condition (2) is satisfied by demanding the relation

$$
Z_{-}(\lambda)=\frac{Z_{+}(\lambda+\zeta)}{Z_{+}(\zeta)},
$$

which in turn implies

$$
P_{-}(x)=P_{+}(x) \frac{e^{-\zeta x}}{\left\langle e^{-\zeta x}\right\rangle_{+}},
$$


where $\left\langle e^{-\zeta x}\right\rangle_{+}=Z_{+}(\zeta)$. This is one of the central results of this paper. It says us that the FR symmetry (11) is always satisfied by (3) whenever the change of measure (5D) is imposed. By reading $x$ as an energy and $\zeta$ as an inverse temperature, its thermodynamic like-structure is self-evident. Similar transformations were introduced in Refs. [24 26]. The structure of Eq. (5) can also be read as a variation of the change of measure used in umbrella sampling [27].

Note that in Eq. (5D) no conditions are required on $P_{+}(x)$, while $P_{-}(x)$ is determined from it. Alternatively, one can choose $P_{-}(x)$ as the independent distribution. In fact, Eq. (2) can also be satisfied by taking

$$
Z_{+}(\lambda)=\frac{Z_{-}(\lambda-\zeta)}{Z_{-}(-\zeta)},
$$

delivering the relation

$$
P_{+}(x)=P_{-}(x) \frac{e^{+\zeta x}}{\left\langle e^{+\zeta x}\right\rangle_{-}},
$$

where $\left\langle e^{+\zeta x}\right\rangle_{-}=Z_{-}(-\zeta)$. These last two expressions follow straightforwardly by inverting Eqs. (4) and (5). Nevertheless, what is important to realize is that, in general, both kind of solutions lead to different family of solutions if one assume the same property for each "independent" distribution.

The relations (5) and (7) do not involves time. Therefore, the proposal Eq. (3) guarantees the fulfillment of the symmetry (11) at any time.

\section{Time-average variables}

When studying a given nonequilibrium steady state, the stochastic variable of interest may be defined through a time average,

$$
\mu_{\tau}(t) \equiv \frac{1}{\tau\langle v\rangle} \int_{t}^{t+\tau} d t^{\prime} v_{s t}\left(t^{\prime}\right)=\frac{x_{s t}(t+\tau)-x_{s t}(t)}{\tau\langle v\rangle},
$$

where $(d / d t) x_{s t}(t)=v_{s t}(t)$. This definition makes sense when the average of the integral contribution grows linearly with time. For example, $x_{s t}(t)$ may represents entropy production and $v_{s t}(t)$ its rate [22], or respectively the position and velocity of a self-propelled particle 23]. $\langle v\rangle$ is the stationary mean value of $v_{s t}(t)$.

Usually the regime of interest is the stationary one, $t=\infty$. Hence, we write $\mu_{\tau}=\lim _{t \rightarrow \infty} \mu_{\tau}(t)$. For this stationary variable, instead of Eq. (11), we write the FR

$$
P\left(-\mu_{\tau}\right)=P\left(\mu_{\tau}\right) \exp \left[-\alpha \mu_{\tau} \tau\right] .
$$

This condition can also be satisfied in the present approach because the statistical properties of $\mu_{\tau}$ follows from those of the stationary increments of $x_{s t}(t)$, that is $x_{\tau}^{\infty} \equiv \lim _{t \rightarrow \infty}\left[x_{s t}(t+\tau)-x_{s t}(t)\right]$. By writing $x_{\tau}^{\infty}$ as the subtraction of two independent stochastic variables related by the change of measures (5) [or (7)], it follows $P\left(-x_{\tau}^{\infty}\right)=\exp \left[-\zeta x_{\tau}^{\infty}\right] P\left(x_{\tau}^{\infty}\right)$. The connection between $x_{\tau}^{\infty}$ and $\mu_{\tau}$ implies the probabilities relation $P\left(\mu_{\tau}\right) d \mu_{\tau}=P\left(x_{\tau}^{\infty}\right) d x_{\tau}^{\infty}$ [28], which lead to FR Eq. (9) with $\alpha=\zeta\langle v\rangle$. Consequently, the proposal based on independent stochastic contributions, as in the previous case [Eq. (10], allows to fulfill the symmetry (9) for any value of $\tau$.

\section{Large deviation functions}

In general, a (rate) variable [Eq. (8)] that characterizes a given non-equilibrium state only satisfies the relation (9) in a long time regime, $\tau \rightarrow \infty$. In fact, the GallavotiCohen FR is an asymptotic relation in time [7 9]. In this regime, when the probability distribution adopt the asymptotic structure $\left(\lim _{\tau \rightarrow \infty}\right) P\left(\mu_{\tau}\right) \approx \exp \left[-\tau \varphi\left(\mu_{\tau}\right)\right]$, the statistics can be analyzed through a large deviation theory [24]. Consistently, the generating function $Z(\lambda)=$ $\left\langle e^{-\lambda \tau \mu_{\tau}}\right\rangle$ scales in the same way, $Z(\lambda) \approx \exp [-\tau \Theta(\lambda)]$. Both $\varphi\left(\mu_{\tau}\right)$ and $\Theta(\lambda)$ define the LDF's of the problem. They completely characterize the asymptotic regime. In terms of $\varphi\left(\mu_{\tau}\right)$, the FR reads

$$
\frac{1}{\tau} \log \left[\frac{P\left(+\mu_{\tau}\right)}{P\left(-\mu_{\tau}\right)}\right] \approx-\varphi\left(+\mu_{\tau}\right)+\varphi\left(-\mu_{\tau}\right)=\alpha \mu_{\tau} .
$$

Through a sadle-point approximation, both LDF can be related by a Legendre-Fenchel transformation [24]

$$
\varphi\left(\mu_{\tau}\right)=\max _{\lambda}\left[\Theta(\lambda)-\lambda \mu_{\tau}\right], \quad \Theta(\lambda)=\min _{\mu_{\tau}}\left[\varphi\left(\mu_{\tau}\right)+\lambda \mu_{\tau}\right] .
$$

These relations and Eq. (10) lead to the equivalent formulation of the FR symmetry [9]

$$
\Theta(\lambda)=\Theta(-\lambda+\alpha) .
$$

Conditions (10) and (12) are well known expressions of Gallavoti-Cohen FR symmetry [7-9]. As the independent variables splitting [Eq. (3)] allows to fulfill the FR (9) at any time, trivially it can also be utilized in the long time regime. After explicitly writing $\mu_{\tau}=\mu_{\tau}^{+}-\mu_{\tau}^{-}$, Eq. (4) and the asymptotic structure of $Z(\lambda)$ allows us to write

$$
\Theta(\lambda)=\Theta_{+}(\lambda)+\Theta_{+}(-\lambda+\alpha)-\Theta_{+}(\alpha),
$$

where $\Theta_{+}(\lambda)$ is the LDF corresponding to the generating function of $\mu_{\tau}^{+}$. By knowing (an arbitrary) $\Theta_{+}(\lambda)$ the previous expression defines $\Theta(\lambda)$, which in turn through Eq. (11) provides the LDF $\varphi\left(\mu_{\tau}\right)$. By construction, the fulfillment of conditions (10) and (12) is guaranteed. Alternatively, from Eq. (6) we can also write

$$
\Theta(\lambda)=\Theta_{-}(-\lambda)+\Theta_{-}(\lambda-\alpha)-\Theta_{-}(-\alpha),
$$

where now $\Theta_{-}(\lambda)$ is the LDF associated to the generating function of $\mu_{\tau}^{-}$. The relations (13) and (14) define the second main result of this paper. They allow to characterizing the long time regime in terms of asymptotic properties of the independent stochastic contributions. 


\section{INTERMITTENT VARIABLES}

Our results allows to build up a variable that satisfy the FR Eq. (9) after knowing the statistical properties of an arbitrary one. The regime of interest is the stationary one, where the FR symmetry is characterized by Eq. (13) or (14). For example one can assume Gaussian or Poissonian statistics. In this last case, if one take $\Theta_{+}(\lambda)=\gamma\left(1-e^{-\lambda}\right)$, which correspond to the LDF of an unidirectional Poisson process with rate $\gamma$, from Eq. (13) we get $\Theta(\lambda)=\gamma\left(1-e^{-\lambda}\right)+\gamma e^{-\alpha}\left(1-e^{\lambda}\right)$. We note that this expression corresponds to the LDF for the entropy production of an asymmetric random walk [22]. Hence, while the previous analysis seem to be rather abstract, there exist non-trivial dynamics where they apply.

The random walk model is able to fit some nonGaussian properties found in Ref. 22. Here, motivated by the experimental results of Ref. [23], we introduce a similar generalized model able to develop intermittence. On the basis of previous analysis, the velocity [see Eq. (8)] is defined as $v_{s t}(t)=v_{s t}^{+}(t)-v_{s t}^{-}(t)$, where

$$
v_{s t}^{ \pm}(t)=x_{0} \sum_{i} \delta\left(t-t_{i}^{ \pm}\right)
$$

The constant $x_{0}$ introduces the right units of $v_{s t}(t), \delta(t)$ is the Dirac delta function, and $t_{i}^{ \pm}$are successive random times. Consistently, $x_{s t}(t)=x_{s t}^{+}(t)-x_{s t}^{-}(t)$,

$$
x_{s t}^{ \pm}(t)=x_{0} n_{s t}^{ \pm}(t),
$$

where $n_{s t}^{ \pm}(t)$ is the number of ( \pm delta) events in the interval $(0, t)$. Hence, they are positive (discrete) random variables. Their generating functions are denoted as $Z_{n}^{ \pm}(s, t)=\left\langle e^{-s n_{s t}^{ \pm}(t)}\right\rangle=\sum_{m=0}^{\infty} q_{m}^{ \pm}(t) e^{-s m}$, where $\left\{q_{m}^{ \pm}(t)\right\}_{m=0}^{\infty}$ are the respective counting probabilities. Independently of their statistics, by choosing $n_{s t}^{+}(t)$ as the "free" variable, this model satisfy the FR symmetry (9) after demanding [see Eq. (5)] the change of measures

$$
q_{m}^{-}(t)=q_{m}^{+}(t) \frac{e^{-s_{0} m}}{\left\langle e^{-s_{0} n_{s t}^{+}(t)}\right\rangle},
$$

where $\left\langle e^{-s_{0} n_{s t}^{+}(t)}\right\rangle=Z_{n}^{+}\left(s_{0}, t\right)$, and $s_{0}$ is an arbitrary positive dimensionless constant. Hence, $n_{s t}^{-}(t)$ can be read as the " $s_{0}$-ensemble" associated to $n_{s t}^{+}(t)$ [26]. After a change of variables based on Eqs. (8) and (16), the constant $\alpha$ reads

$$
\alpha=s_{0} \delta I \equiv s_{0}\left(I^{+}-I^{-}\right),
$$

where $I^{ \pm} \equiv-\left.\lim _{t \rightarrow \infty} t^{-1}(\partial / \partial s) Z_{n}^{ \pm}(s, t)\right|_{s=0}$, or equivalently $\lim _{t \rightarrow \infty}\left\langle n_{s t}^{ \pm}(t)\right\rangle \simeq I^{ \pm} t$, which in turn implies $\langle v\rangle=x_{0}\left(I^{+}-I^{-}\right)$.

In order to close the model, it is necessary to specify the statistical properties of $n_{s t}^{+}(t)$. Its generating function is written as

$$
Z_{n}^{+}(s, t)=Z_{A}^{+}(s, t)+Z_{I}^{+}(s, t)
$$
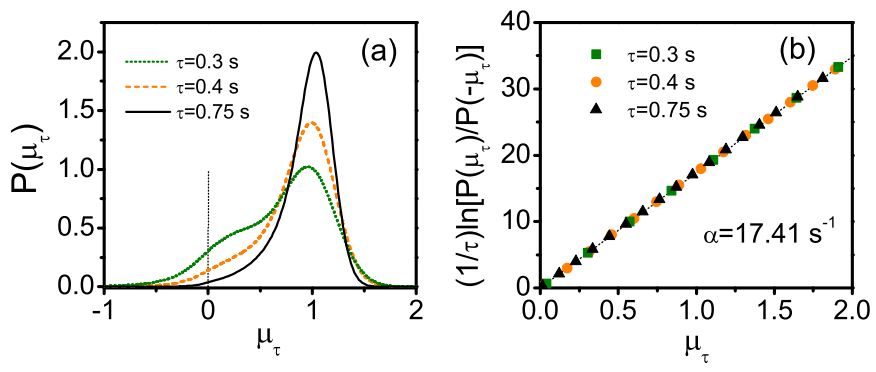

FIG. 1: (color online) (a) Probability distribution $P\left(\mu_{\tau}\right)$ that follows from Eq. (20) (see text) for different values of $\tau$. (b) Linear dependence of $(1 / \tau) \ln \left[P\left(+\mu_{\tau}\right) / P\left(-\mu_{\tau}\right)\right]$ corresponding to the distributions shown in (a). The value of the slope $\alpha$ was obtained from Eq. (18). The parameters [Eq. (20)] are $s_{0}=0.2, \gamma=100 s^{-1}, \Gamma_{A}=0.54 s^{-1}$, and $\Gamma_{I}=4 s^{-1}$.

where the evolution of each contribution read

$$
\begin{aligned}
& \frac{d Z_{A}^{+}(s, t)}{d t}=-\theta_{s} Z_{A}^{+}(s, t)-\Gamma_{A} Z_{A}^{+}(s, t)+\Gamma_{I} Z_{I}^{+}(s, t) \\
& \frac{d Z_{I}^{+}(s, t)}{d t}=+\Gamma_{A} Z_{A}^{+}(s, t)-\Gamma_{I} Z_{I}^{+}(s, t)
\end{aligned}
$$

with $\theta_{s} \equiv \gamma\left(1-e^{-s}\right)$. These dynamics allow us to read $n_{s t}^{+}(t)$ as a modulated Poissonian (counting) process [26], whose rate at random times adopts the values $\gamma_{A}=\gamma$ (Active regime) and $\gamma_{I}=0$ (Inactive regime). The switching between both states is governed by a classical master equation with transition rates $\Gamma_{A}$ and $\Gamma_{I}$. The asymmetric random walk model [22] is recovered from Eq. (20) by taking $\Gamma_{A / I}=0$.

The generating function $Z_{n}^{-}(s, t)$ can be obtained from Eq. (44) after knowing $Z_{n}^{+}(s, t)$. Hence, the (lattice) distribution [28] of the process $x_{s t}(t)$ can be obtained by finding $Z_{n}^{+}(s, t)$ from Eq. (20) and a posterior numerical Fourier inversion of $Z_{n}(s, t)=Z_{n}^{+}(s, t) Z_{n}^{-}(-s, t)$ in the $s=-i k$ variable. By using the Markovian property of Eq. (20), the probability of $x_{\tau}^{\infty} \equiv \lim _{t \rightarrow \infty} x_{s t}(t)$ follows from that of $x_{s t}(t)$ by taking stationary initial conditions for the rate fluctuations. The distribution $P\left(\mu_{\tau}\right)$ of $\mu_{\tau}$ follows from the change of variables defined by Eq. (8).

In Fig. 1(a) we plot the distribution $P\left(\mu_{\tau}\right)$ for different values of $\tau$. For all times the distributions satisfy the symmetry Eq. (9) [Fig. 1(b)]. As expected, we confirmed that this property is valid for any value of the rate parameters that define the evolution (20). On the other hand, the short time behavior of $P\left(\mu_{\tau}\right)$ strongly depends on the chosen parameters values. Nevertheless, for increasing $\tau$ the distributions becomes similar and, consistently, develop an increasing peak around $\mu_{\tau}=1$. In this regime $[\tau \gtrsim 0.3$ in Fig. 1 (a) $]$ a large deviation theory applies. Hence the problem can be analyzed through the corresponding LDF. 


\section{Large deviations functions}

The long time behavior of $Z_{n}^{+}(s, t)$ completely define the asymptotic statistical properties of the counting process $n_{s t}^{+}(t)$. Before characterizing the LDF functions from it, we notice that in the long time limit some results can be established for $Z_{n}^{-}(s, t)$. As demonstrated in Ref. [26], $n_{s t}^{+}(t)$ can be mapped with a renewal process characterized by a shift closure property, which implies that asymptotically $n_{s t}^{-}(t)$ also becomes a renewal process with a renormalized waiting time distribution. Hence, asymptotically $Z_{n}^{-}(s, t)$ has the same structure and dynamics than $Z_{n}^{+}(s, t)$ but with renormalized rates. After some hard calculations steps, which are not relevant for the following analysis, we obtained $\gamma \rightarrow \gamma_{s_{0}}=\gamma e^{-s_{0}}$, and more complex expressions for the hopping rates $\Gamma_{A / I} \rightarrow \Gamma_{A / I}\left(s_{0}\right)$. Furthermore $I^{+}=\gamma \Gamma_{I} /\left(\Gamma_{A}+\Gamma_{I}\right)$, and similarly $I^{-}=\gamma_{s_{0}} \Gamma_{I}\left(s_{0}\right) /\left[\Gamma_{A}\left(s_{0}\right)+\Gamma_{I}\left(s_{0}\right)\right]$. In the case of an asymmetric random walk model [22], $\Gamma_{A / I}=0$, both set of probabilities $\left\{q_{m}^{ \pm}(t)\right\}_{m=0}^{\infty}$ become Poissonian counting processes with $I^{+}=\gamma$, and $I^{-}=\gamma e^{-s_{0}}$.

By working Eqs. (19) and (20) in a Laplace domain, $f(\xi)=\int_{0}^{\infty} f(t) e^{-\xi t}$, it is possible to write $Z_{n}^{+}(s, t)$ as a superposition of two exponential functions scaled by the roots $[Q(\xi)=0]$ of the characteristic polynomial

$$
Q(\xi)=\xi^{2}+\xi\left(\theta_{s}+\Gamma_{A}+\Gamma_{I}\right)+\theta_{s} \Gamma_{I} .
$$

From its definition, the smaller solution [22], after the change of variable $s \rightarrow \lambda / \delta I$, provides $\Theta_{+}(\lambda)$. We get

$\Theta_{+}(\lambda)=\frac{\theta_{\lambda}^{\prime}+\Gamma_{A}+\Gamma_{I}}{2}-\left[\Gamma_{A} \theta_{\lambda}^{\prime}+\frac{1}{4}\left(\Gamma_{A}+\Gamma_{I}-\theta_{\lambda}^{\prime}\right)^{2}\right]^{1 / 2}$,

where $\theta_{\lambda}^{\prime} \equiv \theta_{\lambda / \delta I}=\gamma\left(1-e^{-\lambda / \delta I}\right)$. This result, joint with Eq. (13) and the transformation (11) completely characterize the asymptotic statistics and LDF of $\mu_{\tau}$.

In Fig. 2(a) we plot the LDF $\Theta(\lambda)$ for two different set of parameter values $\left\{s_{0}, \gamma, \Gamma_{A}, \Gamma_{I}\right\}$. In both cases the symmetry (12) is satisfied. The values of $\alpha$ were determined from (18). Notice that $\Theta(\lambda)$ cannot be well approximated by a quadratic polynomial, which in turn implies the presence of strong non-Gaussian features. Figs. 2 (b) and 2(c) confirm this fact. Both LDF $\varphi\left(\mu_{\tau}\right)$ were obtained numerically through the Legendre transformation (11). The same results follows from the asymptotic behavior of the exact distribution $P\left(\mu_{\tau}\right)$ obtained by Fourier inversion of its generating function. Consistently, the LDF and the asymptotic exact distribution satisfy the FR (10). In the insets, we plot the time dependence of the skewness $S=\left\langle\delta \mu_{\tau}^{3}\right\rangle / \sigma^{3}$ and kurtosis $K=\left\langle\delta \mu_{\tau}^{4}\right\rangle / \sigma^{4}-3$, obtained from the generating function of $P\left(\mu_{\tau}\right)$, where $\delta \mu_{\tau}=\mu_{\tau}-\left\langle\mu_{\tau}\right\rangle$ and $\sigma^{2}=\left\langle\delta \mu_{\tau}^{2}\right\rangle$. These objects also confirm the strong non-Gaussian nature of $P\left(\mu_{\tau}\right)$.

The functions $\varphi\left(\mu_{\tau}\right)$ not only depart from a quadratic polynomial, but also develop a "kink," that is, and abrupt change around the origin. This characteristic arises when $\Theta(\lambda)$ presents a plateau regime cen-
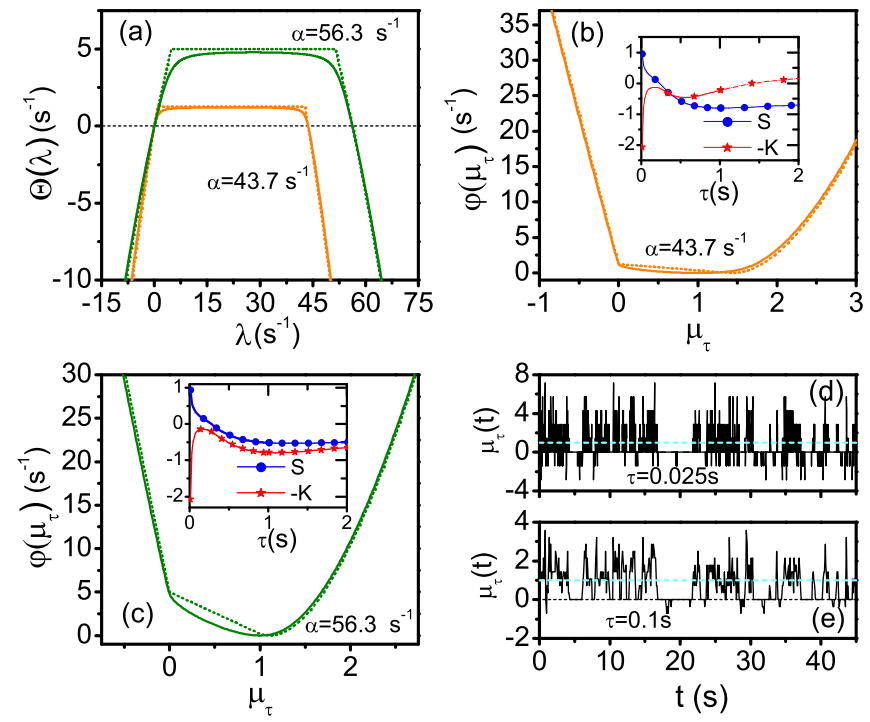

FIG. 2: (color online) (a) LDF $\Theta(\lambda)$ [Eq. (13)] for two different values of $\alpha$. (b)-(c) $\operatorname{LDF} \varphi\left(\mu_{\tau}\right)$ [Eq. (11)] for $\alpha=43.7 \mathrm{~s}^{-1}$ and $\alpha=65.5 \mathrm{~s}^{-1}$ respectively. (d)-(e) Realizations of $\mu_{\tau}(t)$ $\left[\alpha=43.7 \mathrm{~s}^{-1}\right]$ for $\tau=0.025 \mathrm{~s}$ and $\tau=0.1 \mathrm{~s}$ respectively. In all cases, the dotted lines follows from approximation (23). The insets show the skewness $(\mathrm{S})$ and kurtosis $(\mathrm{K})$ as function of $\tau$ indicating the strongly non-Gaussian nature of $\mu_{\tau}$. The parameters [Eq. (20)] for the curves with $\alpha=43.7 \mathrm{~s}^{-1}$ are $s_{0}=1.56, \gamma=40 s^{-1}, \Gamma_{A}=0.54 s^{-1}$, and $\Gamma_{I}=1.25 s^{-1}$. For $\alpha=56.3 s^{-1}$ are the same except $\Gamma_{I}=5 s^{-1}$.

tered around $\alpha / 2$. For the chosen parameters values, the curves and these special features are similar [29] to those found in the numerical and experimental results of Refs. [22, 23]. In the present model, a physical effect can be associated to these properties: the kink and the plateau regime are closely related with the development of intermittence in the stochastic realizations of $\mu_{\tau}$. This is the third main result of this contribution.

The stochastic realizations of $n_{s t}^{+}(t)$ can be obtained from Eq. (20) by using standard monte Carlo methods. Alternatively, a more simple algorithm follows by mapping Eq. (20) with a renewal process 26]. On the other hand, the realizations of $n_{s t}^{-}(t)$ can be obtained from a conditional scheme defined in Ref. [26], where realizations of $n_{s t}^{+}(t)$ with $m$-events are selected as one of $n_{s t}^{-}(t)$ with probability $e^{-s_{0} m}$ and discarded with probability $\left(1-e^{-s_{0} m}\right)$. Statistically independent realizations of $n_{s t}^{ \pm}(t)$ allow to generate the stochastic trajectories of $\mu_{\tau}$ [see Eqs. (8) and (16)].

In Figs. 2(d) and (e) we plot two realizations of $\mu_{\tau}$, corresponding to $\alpha=43.7 \mathrm{~s}^{-1}$, for two different values of $\tau$. Consistently with the definition (8) they fluctuates around $\mu_{\tau}=1$. For the chosen parameter values, the trajectories switch amongst periods of time where $\mu_{\tau}$ is positive, negative or null. As expected, for increasing $\tau$ the fluctuations are diminished. Furthermore, for higher averaging times (not shown) the realizations only assume 

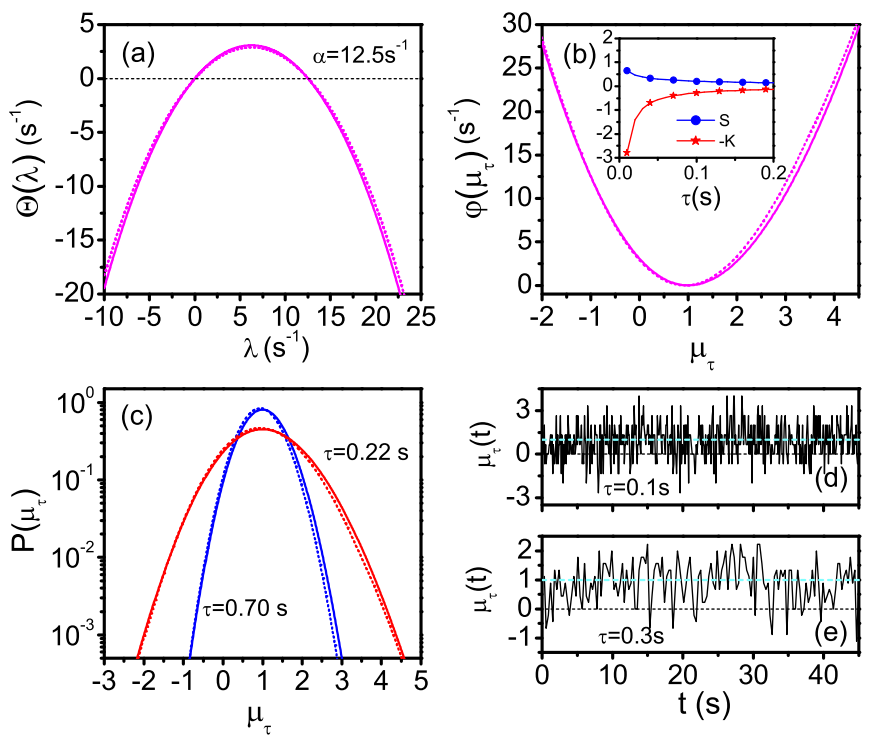

FIG. 3: (color online) (a) LDF $\Theta(\lambda)$ [Eq. (13)] with $\alpha=$ $12.5 s^{-1}$. (b) $\operatorname{LDF} \varphi\left(\mu_{\tau}\right)$ [Eq. (11)]. (c) Probability distribution of $\mu_{\tau}$ for $\tau=0.22 \mathrm{~s}$ and $\tau=0.70 \mathrm{~s}$. (d)-(e) Realizations of $\mu_{\tau}(t)$ for $\tau=0.1 \mathrm{~s}$ and $\tau=0.3 \mathrm{~s}$ respectively. Inset show the skewness (S) and kurtosis (K) as function of $\tau$ indicating tendency to become more Gaussian at higher time $\tau$. In all curves the parameters [Eq. (20)] are $s_{0}=0.83, \gamma=25 \mathrm{~s}^{-1}$, $\Gamma_{A}=0.3 s^{-1}, \Gamma_{I}=20 s^{-1}$. The dotted lines follows from approximation (23).

positive values. From Eq. (20), it is immediate to deduce that when $\gamma \gg\left(\Gamma_{A}, \Gamma_{I}\right)$, the realizations of $n_{s t}^{+}(t)$ develop intermittence. This is not a surprising result. What is novel and not trivial, is that one can build up the complementary process $n_{s t}^{-}(t)$ such that their (normalized) subtraction [Eq. (8)] satisfy the FR. The closeness between the kinks of Figs. 2(b) and (c) and the intermittence shown in Figs. 2(d) and (e) is also supported by the results shown in Fig. 3. Here, $\Gamma_{I} \gg \Gamma_{A}$. Therefore, the inactive regime is statistically (dynamically) inhibited, which in turn implies that neither $n_{s t}^{+}(t)$ or $n_{s t}^{-}(t)$ develops any intermittence. As shown by the plots, the LDF converge to quadratic polynomial functions, while the probability distribution becomes Gaussian. Consistently, the trajectories, Figs. 3(d) and (e), for any value of $\tau$ do not develop the phenomenon of intermittence.

Eqs. (13) and (22) provide an analytical expression for the $\operatorname{LDF} \Theta(\lambda)$ that is very complicated. Furthermore, the transformation Eq. (11), which delivers the LDF $\varphi\left(\mu_{\tau}\right)$, is only manageable numerically. Nevertheless, when $\Gamma_{A} \ll\left(\gamma, \Gamma_{I}\right)$, from Eq. (21) it is simple to deduce the expression

$$
\lim _{\Gamma_{A} \rightarrow 0} \Theta_{+}(\lambda)=\min \left[\theta_{\lambda}^{\prime}, \Gamma_{I}\right]
$$

This result, joint with Eqs. (11) and (13) allow us to obtain simple analytical expressions for the $\operatorname{LDF} \Theta(\lambda)$ and $\varphi\left(\mu_{\tau}\right)$ (see Appendix). They correspond to the dotted lines of Figs. 2 and 3. Evidently, they provide a very good fitting to the plotted curves. Nevertheless, what is more relevant is the possibility of getting an analytical description of the relation between intermittence and the non-Gaussian features of both LDF.

In the limit $\Gamma_{A} \rightarrow 0$, both $\Theta(\lambda)$ and $\varphi\left(\mu_{\tau}\right)$ present points where they are not derivable functions with respect to their arguments. In particular, when intermittence develops, $\Gamma_{I} \ll \gamma$, the $\operatorname{LDF} \varphi\left(\mu_{\tau}\right)$ has a linear behavior around the origin with different slopes for $\mu_{\tau} \lessgtr 0$ [see dotted lines $\left(\mu_{\tau} \gtrless 0\right)$ in Figs. 2(b) an (c)]. The addition of the slopes of $\varphi\left(\mu_{\tau}\right)$ around the origin is equal to $-\alpha$ [see Eq. (A.12)]. The experimental data of Ref. [23] seem to be consistent with this relation. On the other hand, in the intermittence regime $\Theta(\lambda)$ develops a plateau regime [see dotted lines in Figs. 2(a)] with value $\Gamma_{I}$ [Eq. A.11], which is similar to that found in the numerical results of Ref. 22].

Finally, let us remark that the discontinuities of the derivative of $\Theta(\lambda)$ can be read as phase transitions between different dynamical regimes of $\mu_{\tau}$ [25, 26]. These features, as well as our main proposal, Eq. (5), suggest an interesting bridge between FR and equilibrium thermodynamics.

\section{CONCLUSIONS}

In conclusion, we have demonstrated that FR are always satisfied by the subtraction of two independent stochastic variables whose probability distributions are related by a thermodynamic-like change of measure. This relation leaves completely arbitrary one of the distributions, being this degree of freedom the basis of the present approach. By choosing one of then as a random modulated Poisson process, the distribution of interest develops strong non-Gaussian features, which in turn arise in the parameter regime where intermittence develops. Hence, variables such as entropy production rates may satisfy FR and develop intermittence.

Depending of the parameters values of the model the LDF develop a rich variety of functional dependences, which in turn are similar to those found in recent numerical and experimental results [29]. In particular, in the intermittence regime the LDF of the probability distribution and generating function are characterized by a kink at the origin and a plateau regime respectively. While our analysis does not relies on any specific (non-linear many body) dynamical model, it strongly suggest that intermittence may be a central ingredient in nonequilibrium steady states characterized by non-Gaussian fluctuations.

Due to the wide class of systems where intermittence develops, added to the central role of FR in nonequilibrium states, the characterization of dynamical conditions that guaranty the coexistence of these two properties becomes a very interesting open issue, for which this paper intends to contribute. On other hand, the inclusion of statistical correlations that preserve the FR in the two-variables model as well as the analysis of these 
ideas in the context of deterministic thermostated systems (phase space contraction conjecture) 13] are additional open issues that may deserve extra analysis.

\section{Acknowledgments}

This work was supported by CONICET, Argentina, PIP 11420090100211.

\section{Appendix: LDF in the limit $\Gamma_{A} \rightarrow 0$}

Here we obtain the LDF associated to Eq. (23). $\Theta(\lambda)$ follows from Eq. (13). Hence, $\varphi\left(\mu_{\tau}\right)$ is obtained through the Legendre transformation (11). In order to simplify the expressions, we write

$$
\Theta(\lambda)=\bar{\Theta}(s=\lambda / \delta I),
$$

and similarly $\varphi\left(\mu_{\tau}\right)$ as

$$
\varphi\left(\mu_{\tau}\right)=\bar{\varphi}\left(k=\mu_{\tau} \delta I\right),
$$

where $\delta I=s_{0}\left(I^{+}-I^{-}\right)$[Eq. (18)]. The functions $\bar{\Theta}(s)$, and $\bar{\varphi}(k)$ are the LDF of the stochastic variable $k_{s t}(t)=$ $\left.n_{s t}^{+}(t)-n_{s t}^{-}(t)\right] / t$. Furthermore, we define the parameters

$$
\begin{aligned}
& s^{+} \equiv-\ln \left(1-\frac{\Gamma_{I}}{\gamma}\right), \\
& s^{-} \equiv s_{0}+\ln \left(1-\frac{\Gamma_{I}}{\gamma}\right),
\end{aligned}
$$

and respectively

$$
\begin{aligned}
& k^{+} \equiv \gamma e^{-s^{+}}=\gamma\left(1-\frac{\Gamma_{I}}{\gamma}\right), \\
& k^{-} \equiv \gamma e^{-s^{-}}=\gamma \frac{e^{-s_{0}}}{1-\frac{\Gamma_{I}}{\gamma}} .
\end{aligned}
$$

The LDF of a Poisson process with rate $\gamma$ is denoted as

$$
\theta(s) \equiv \gamma\left(1-e^{-s}\right),
$$

and its (symmetrized) Legendre transform as

$$
\varphi_{p}(k) \equiv \gamma\left\{1-\frac{|k|}{\gamma}\left[1-\ln \left(\frac{|k|}{\gamma}\right)\right]\right\} .
$$

Finally, we introduce the function

$$
\begin{aligned}
\varphi_{r w}(k) \equiv & \gamma\left\{1+e^{-s_{0}}-\left(4 e^{-s_{0}}+\frac{k^{2}}{\gamma^{2}}\right)^{1 / 2}\right. \\
& \left.+\frac{k}{\gamma} \ln \left[\frac{1}{2}\left(4 e^{-s_{0}}+\frac{k^{2}}{\gamma^{2}}\right)^{1 / 2}+\frac{k}{2 \gamma}\right]\right\} .
\end{aligned}
$$

Both $\bar{\Theta}(s)$ and $\bar{\varphi}(k)$ must be defined in different parameter regimes. i) In the parameter regime

$$
0<\frac{\Gamma_{I}}{\gamma}<\left(1-e^{-s_{0} / 2}\right),
$$

it follows $s^{+}<s^{-}$, with

$$
\bar{\Theta}(s)=\left\{\begin{array}{cc}
\theta(s), & s<s^{+} \\
\Gamma_{I}, & s^{+}<s<s^{-} \\
\theta\left(-s+s_{0}\right), & s^{-}<s
\end{array}\right.
$$

and respectively

$$
\bar{\varphi}(k)=\left\{\begin{array}{cc}
\varphi_{p}(k)-k s_{0}, & k<-k^{+}, \\
\Gamma_{I}-k s^{-}, & -k^{+}<k<0, \\
\Gamma_{I}-k s^{+}, & 0<k<k^{+}, \\
\varphi_{p}(k), & k^{+}<k .
\end{array}\right.
$$

This is one of the more interesting parameter regimes, which in fact corresponds to the intermittence one (Fig. $2)$. Notice that in the plateau regime of $\Theta(\lambda)\left(s^{+} \delta I<\right.$ $\left.\lambda<\delta I s^{-}\right)$it assumes the value $\Gamma_{I}$. On the other hand, around the origin $\varphi\left(\mu_{\tau}\right)$ has a linear behavior which different slopes for $\mu_{\tau} \gtrless 0$. This property gives rise to the characteristic kink shown in Figs. 2(b) and (c). From Eqs. A.2 and (A.12), we deduce that the addition of the slopes is equal to $-\alpha$ [Eq. (18)]. Furthermore, from Eq. A.12, it is possible to demonstrate that the origin is the unique point at which the derivative of $\varphi\left(\mu_{\tau}\right)$ is a discontinuous function. Remarkably, in the following parameter regimes $\varphi\left(\mu_{\tau}\right)$ has a continuous derivative. Hence, a kink related to a discontinuous derivative only arises in the present case.

ii) In the parameter regime

$$
\left(1-e^{-s_{0} / 2}\right)<\frac{\Gamma_{I}}{\gamma}<\left(1-e^{-s_{0}}\right),
$$

it follows $s^{-}<s^{+}$, with

$$
\bar{\Theta}(s)=\left\{\begin{array}{cc}
\theta(s), & s<s^{-}, \\
\theta(s)+\theta\left(-s+s_{0}\right)-\Gamma_{I}, & s^{-}<s<s^{+}, \\
\theta\left(-s+s_{0}\right), & s^{+}<s,
\end{array}\right.
$$

and by defining the parameters

$$
k^{*} \equiv k^{-}-k^{+}, \quad \Delta \equiv \Gamma_{I}-\theta\left(s_{0}\right),
$$

$\left(k^{*}>0\right)$, we write

$$
\bar{\varphi}(k)=\left\{\begin{array}{cc}
\varphi_{p}(k)-k s_{0}, & k<-k^{-}, \\
\left(\gamma-k^{-}\right)-k s^{+}, & -k^{-}<k<-k^{*}, \\
\varphi_{r w}(k)-\Delta, & -k^{*}<k<k^{*}, \\
\left(\gamma-k^{-}\right)-k\left(s_{0}-s^{+}\right), & k^{*}<k<k^{-}, \\
\varphi_{p}(k), & k^{-}<k .
\end{array}\right.
$$

In this intermediate regime, the non-Gaussian properties are gradually lost. The plateau regime of $\Theta(\lambda)$ becomes bend. On the other hand, we remark that even when $\varphi\left(\mu_{\tau}\right)$ is defined by parts, its first derivative is a continuous function. 
iii) In the parameter regime

$$
\left(1-e^{-s_{0}}\right)<\frac{\Gamma_{I}}{\gamma}<1
$$

with $s^{-}<s^{+}$, we get

$$
\bar{\Theta}(s)=\left\{\begin{array}{cc}
\theta(s)+\Delta, & s<s^{-}, \\
\theta(s)+\theta\left(-s+s_{0}\right)-\theta\left(s_{0}\right), & s^{-}<s<s^{+}, \\
\theta\left(-s+s_{0}\right)+\Delta, & s^{+}<s,
\end{array}\right.
$$

and

$$
\bar{\varphi}(k)=\left\{\begin{array}{cc}
\varphi_{p}(k)-k s_{0}+\Delta, & k<-k^{-}, \\
\left(\gamma-k^{-}\right)-k s^{+}+\Delta, & -k^{-}<k<-k^{*}, \\
\varphi_{r w}(k), & -k^{*}<k<k^{*}, \\
\left(\gamma-k^{-}\right)-k\left(s_{0}-s^{+}\right)+\Delta, & k^{*}<k<k^{-}, \\
\varphi_{p}(k)+\Delta, & k^{-}<k .
\end{array}\right.
$$

Fig. 3 falls in this regime, where both LDF approach quadratic functions. In fact, while the derivative of $\Theta(\lambda)$ presents smooth discontinuities they are far beyond of the origin $\left[\lim _{\left(\Gamma_{I} / \gamma\right) \rightarrow 1} s^{ \pm}= \pm \infty\right]$. As in the previous case, $\varphi\left(\mu_{\tau}\right)$ has a continuous derivative. Its dependence is mainly defined by the function $\varphi_{r w}(k)\left[\lim _{\left(\Gamma_{I} / \gamma\right) \rightarrow 1} k^{*}=\right.$ $\infty]$.

iv) In the parameter regime

$$
1<\frac{\Gamma_{I}}{\gamma}<\infty
$$

for any value of $s$, we get

$$
\bar{\Theta}(s)=\theta(s)+\theta\left(-s+s_{0}\right)-\theta\left(s_{0}\right) .
$$

Therefore, in this approximation $\Theta(\lambda)$ correspond to the LDF of an asymmetric random walk. Furthermore, it follows $\bar{\varphi}(k)=\varphi_{r w}(k)$ [Eq. A.9 $]$. As in the previous regime, the rate fluctuations do not induce any nonGaussian feature because their characteristic time is comparable to that of the individual events.
[1] N. Platt, E.A. Spiegel, and C. Tresser, Phys. Rev. Lett. 70, 279 (1993); C. Grebogi, E. Ott, F. Romeiras, and J.A. Yorke, Phys. Rev. A 36, 5365 (1987).

[2] M.B. Plenio and P.L. Knight, Rev. Mod. Phys. 70, 101 (1998).

[3] A.L. Efros and M. Rosen, Phys. Rev. Lett. 78, 1110 (1997); K.T. Shimizu, R.G. Neuhauser, C.A. Leatherdale, S.A. Empedocles, W.K. Woo, and M.G. Bawendi, Phys. Rev. B 63, 205316 (2001).

[4] J. Wang and P. Wolynes, Phys. Rev. Lett. 74, 4317 (1995).

[5] P. Bak and K. Sneppen, Phys. Rev. Lett. 71, 4083 (1993).

[6] G. Falkovich, K. Gawedzki, M. Vergasola, Rev. Mod. Phys. 73, 913 (2001).

[7] G. Gallavotti and E.G.D. Cohen, Phys. Rev. Lett. 74, 2694 (1995).

[8] J. Kurchan, J. Phys. A 31, 3719 (1998).

[9] J.L. Lebowitz and H. Spohn, J. Stat. Phys. 95, 333 (1999).

[10] G.E. Crocks, Phys. Rev. E 60, 2721 (1999).

[11] C. Maes, J. Stat. Phys. 95, 367 (1999).

[12] U. Seifert, Phys. Rev. Lett. 95, 040602 (2005).

[13] E.M. Sevick, R. Prabhakar, S.R. Williams, and D.J. Searles, Annu. Rev. Phys. Chem 59, 603 (2008).

[14] P. Gaspard, J. Chem. Phys. 120, 8898 (2004).

[15] W.I. Goldburg, Y.Y. Goldschmidt, and H. Kellay, Phys. Rev. Lett. 87, 245502 (2001).

[16] G.M. Wang, E.M. Sevick, E. Mittag, D.J. Searles, and D.J. Evans, Phys. Rev. Lett. 89, 050601 (2002).

[17] D.M. Carberry, J.C. Reid, G.M. Wang, E.M. Sevick, D.J. Searles, and D.J. Evans, Phys. Rev. Lett. 92, 140601 (2004).

[18] A. Puglisi, P. Visco, A. Barrat, E. Trizac, and F. van Wijland, Phys. Rev. Lett. 95, 110202 (2005).

[19] S. Schuler, T. Speck, C. Tietz, J. Wrachtrup, and U.
Siefert, Phys. Rev. Lett. 94, 180602 (2005).

[20] S. Majumdar and A.K. Sood, Phys. Rev. Lett. 101, 078301 (2008).

[21] M. Belushkin, R. Livi, and G. Foffi, Phys. Rev. Lett. 106, 210601 (2011).

[22] J. Mehl, T. Speck, and U. Seifert, Phys. Rev. E 78, 011123 (2008).

[23] N. Kumar, S. Ramaswamy, and A.K. Sood, Phys. Rev. Lett. 106, 118001 (2011).

[24] H. Touchette, Phys. Rep. 478, 1 (2009).

[25] J.P. Garrahan and I. Lesanovsky, Phys. Rev. Lett. 104, 160601 (2010); A.A. Budini, Phys. Rev. E 82, 061106 (2010).

[26] A.A. Budini, Phys. Rev. E 84, 011141 (2011).

[27] S.R. Williams and D.J. Evans, Phys. Rev. Lett. 105, 110601 (2010); A. Warmflash, P. Bhimalapuram, and A.R. Dinner, J. Chem. Phys. 127, 154112 (2007).

[28] N.G. van Kampen, Stochastic Processes in Physics and Chemistry, (Sec. Ed., North-Holland, Amsterdam, 1992).

[29] Notice that Figs. 2(b), 2(c), and 3(c) are respectively very similar to Figs. 2(c), 2(d) and 3(b) of Ref. 23. In addition, Figs. 1 of Ref. 22] can be recovered with other parameter values. Besides these similitudes, it is important to notice that our analysis only concentrates on asymptotic statistical properties of $\mu_{\tau}$. Hence, there may exist different processes $v(t)$, and $x(t)$, that lead to the same long-time statistical behaviors. On the other hand, the description of specific non-stationary properties, where in general the FR is not satisfied, as well as the relation between the parameters of the model and experimental variables, are interesting open problems whose solutions depend on each specific situation. These issues are beyond the scope of this contribution. 